ज्ञ FRANÇAISE

$\supset$ DE

때 PÉDAGOGIE

\section{Revue française de pédagogie}

Recherches en éducation

162 | janvier-mars 2008

Acquisitions et progressions scolaires : recherches en psychologie

\title{
Évaluation d'entraînements multisensoriels de préparation à la reconnaissance de figures géométriques planes chez les enfants de cinq ans : étude de la contribution du système haptique manuel
}

Assessing multi sensory training activities preparing five-year-old children to recognize plane geometrical figure: study of the contribution of the manual haptic system

Evaluación de entrenamientos multisensoriales de preparación al reconocimiento de figuras geométricas planas en los niños de cinco años : estudio de la contribución del sistema háptico manual Bewertung von multisensoriellen Trainingsübungen zur Vorbereitung bei der Wiedererkennung flacher geometrischer Formen bei fünfjährigen Kindern: der Beitrag des manuellen haptischen Systems

\section{Laëtitia Pinet et Édouard Gentaz}

\section{OpenEdition}

Journals

Édition électronique

URL : http://journals.openedition.org/rfp/753

DOI : $10.4000 /$ rfp.753

ISSN : 2105-2913

Éditeur

ENS Éditions

Édition imprimée

Date de publication : 1 janvier 2008

Pagination : 29-44

ISBN : 978-2-7342-1118-1

ISSN : 0556-7807

Référence électronique

Laëtitia Pinet et Édouard Gentaz, «Évaluation d'entraînements multisensoriels de préparation à la reconnaissance de figures géométriques planes chez les enfants de cinq ans : étude de la contribution du système haptique manuel », Revue française de pédagogie [En ligne], 162 I janvier-mars 2008, mis en ligne le 01 janvier 2012, consulté le 30 avril 2019. URL : http://journals.openedition.org/ rfp/753 ; DOl : 10.4000/rfp.753 
(c) tous droits réservés 


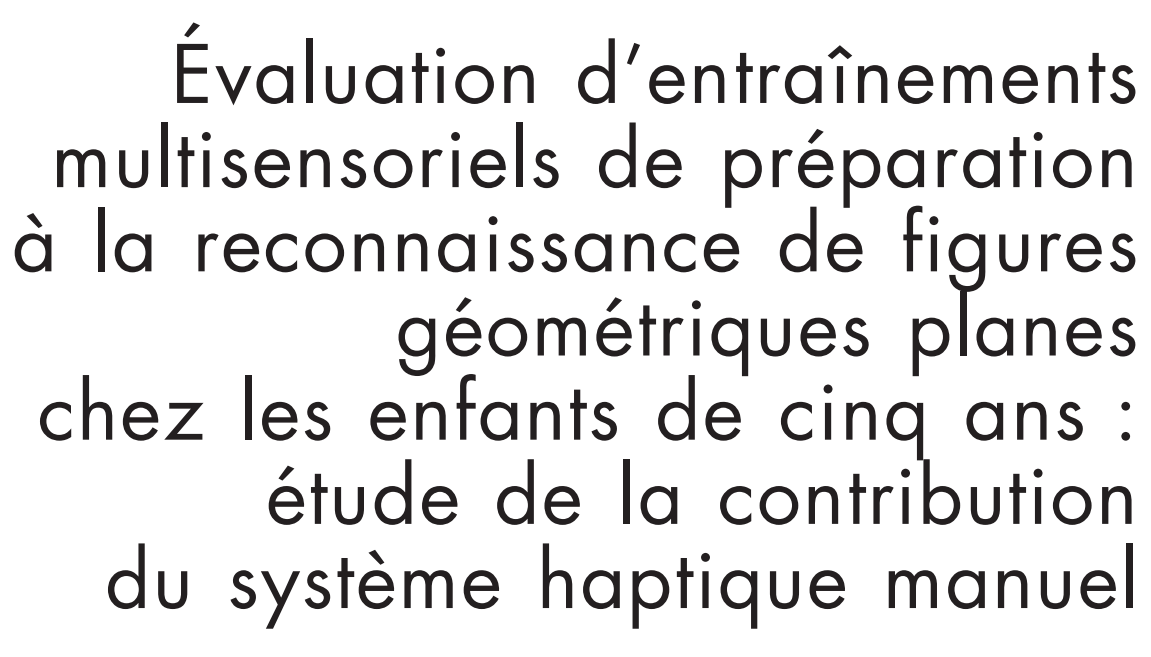

Laëtitia Pinet et Édouard Gentaz

Cette recherche évalue chez trente quatre enfants âgés de cinq ans les effets de l'ajout de la modalité haptique (tactilo-kinesthésique) dans un entraînement destiné à favoriser la reconnaissance visuelle de quatre figures géométriques planes (cercle, carré, rectangle et triangle). Deux entraînements sont évalués. Ils ont en commun de proposer des exercices centrés sur des propriétés de chaque figure, mais ils diffèrent sur les modalités sensorielles sollicitées pour les explorer. Dans l'entraînement «VH » (visuo-haptique), les modalités visuelle et haptique sont utilisées pour explorer les figures en relief alors que seule la modalité visuelle est mobilisée dans l'entraînement « $V$ » (visuel). Les résultats révèlent une amélioration significative des performances seulement après l'entraînement " $V H$ ». Ces résultats sont expliqués par les caractéristiques de la modalité haptique manuelle.

Descripteurs (TEE) : mathématiques, géométrie, main, apprentissage, catégorie.

\section{INTRODUCTION}

Développer les connaissances géométriques et spatiales est un objectif important de l'enseignement des mathématiques à l'école et ce, dès le plus jeune âge, parce qu'elles sont reconnues comme essentielles à l'Homme pour bien interagir avec son environnement. Cette recherche évalue, chez les élèves de grande section de maternelle, les effets de l'ajout de la modalité haptique (tactilo-kinesthésique) dans un entraînement destiné à favoriser la reconnaissance de quatre figures géométriques planes élémentaires : le cercle, le carré, le rectangle et le triangle. Rappelons (Baruk, 1995) que le cercle est communément défini comme une figure plane et fermée dont tous les points sont à égale distance d'un même point appelé centre, le carré est défini comme étant un quadrilatère équilatéral et équiangulaire alors que le rectangle est un quadrilatère équiangulaire uniquement, enfin, le triangle, est un polygone à trois côtés déterminé par trois points non alignés dans un plan.

Ces quatre figures géométriques peuvent être considérées comme quatre catégories comprenant 
une infinité d'exemplaires partageant des propriétés géométriques (Neisser, 1987). Quelques études ont examiné comment les jeunes enfants arrivent à traiter de la même façon, différents exemplaires d'une même catégorie et à dépasser leurs spécificités au profit de leur généralité. Cette activité de catégorisation peut permettre ainsi à l'enfant d'organiser ses connaissances géométriques et de les généraliser à de nouveaux exemplaires. Rappelons que les attentes formulées dans les programmes du ministère français de l'Éducation nationale (2002) mentionnent comme objectif d'amener progressivement les élèves à dépasser une reconnaissance des figures fondées seulement sur leurs apparences visuelles. Plus spécifiquement, parmi les compétences visées à la fin du cycle des apprentissages fondamentaux, les enfants doivent être capables de reconnaître un triangle, un carré, un rectangle, un cercle parmi d'autres figures planes. Les résultats des études menées dans divers pays et à différents niveaux scolaires révèlent que cette acquisition est loin d'être triviale.

Les évaluations réalisées par le ministère français de l'Éducation nationale (2003) auprès d'élèves de CE2, montrent par exemple que les difficultés des enfants proviennent des figures proposées par les tests et de l'influence de certains exemplaires de figures comme les prototypes (cf. plus loin pour une définition). Dans une perspective développementale, D. Clements et al. (1999) étudient la façon dont les enfants de quatre, cinq et six ans reconnaissent les exemplaires d'une catégorie de figure parmi d'autres figures en leur demandant de marquer chaque figure cible. Les quatre tests (un pour chaque figure) sont présentés en annexe 1. Les auteurs mesurent le nombre de figures cibles correctement reconnues parmi les distracteurs. Les résultats révèlent une augmentation des performances avec l'âge, pondérée par le type de figure. Ainsi, le cercle est très bien reconnu à tous les âges : $92 \%$ à quatre ans, $95 \%$ à cinq ans et $99 \%$ à six ans. Le carré est reconnu à $82 \%$ à quatre ans, à $86 \%$ à cinq ans et à $91 \%$ à six ans. Le rectangle est reconnu à $51 \%$ à quatre ans, à $51 \%$ à cinq ans et $59 \%$ à six ans. Enfin, le triangle est reconnu à $57 \%$ à quatre ans, à $58 \%$ à cinq ans et $61 \%$ à six ans. II est cependant difficile de comparer les connaissances des enfants d'une figure à l'autre sans contrôler plus rigoureusement les figures cibles et distractrices proposées. En effet, le nombre de figures cibles et le nombre de figures distractrices ne sont pas les mêmes entre les quatre tests. De plus, le choix des figures (la taille et l'orientation) ne semble pas contrôlé.
En prenant en compte ces remarques, L. Pinet et É. Gentaz (2007) étudient chez quarante quatre enfants âgés de cinq ans la reconnaissance de quatre catégories de figures géométriques planes (cercle, carré, rectangle et triangle) et confirment globalement les résultats de $\mathrm{D}$. Clements et al. (1999). Les enfants passent individuellement quatre tests papier-crayon, un pour chaque catégorie de figure. Chaque feuille-test comprend vingt figures, dont six cibles parmi quatorze distracteurs, présentées dans les annexes $2 a$ et $2 b$. Les résultats révèlent que les difficultés des jeunes enfants varient en fonction de la catégorie et de l'exemplaire de la figure considérée, avec un meilleur taux de reconnaissance pour le cercle (99\%), puis pour le carré $(73 \%)$, puis pour le rectangle $(64 \%)$ et enfin pour le triangle (53\%). De plus, les analyses indiquent que certains exemplaires des trois catégories de figures (carré, rectangle et triangle) sont mieux reconnus : le carré avec un de ses côtés aligné sur l'horizontale, le rectangle possédant un rapport de 1,5 entre son petit côté et son grand côté, ce dernier étant aligné sur l'horizontale en enfin, le triangle équilatéral avec un côté aligné sur l'horizontale. Ces exemplaires particuliers sont considérés comme les "prototypes" de chaque catégorie de figure. De façon complémentaire, l'analyse des erreurs (fausses reconnaissances) montre que leurs nombres ne différent pas significativement pour le carré $(23 \%)$, le rectangle $(18 \%)$ et le triangle $(27 \%)$. Ces résultats suggèrent que la catégorisation des carrés, des rectangles et des triangles n'est pas encore très spécifiée (i.e., ce qui est inclus et ce qui est exclu). L'ensemble des résultats confirme que les enfants de grande section de maternelle commencent à être capables de se représenter les figures géométriques élémentaires mais avec des niveaux qui diffèrent selon la catégorie et l'exemplaire de la figure considérée. En d'autres termes, toutes les figures ainsi que tous les exemplaires d'une même figure ne sont pas reconnus de façon équivalente.

La question est alors d'étudier comment favoriser l'apprentissage des figures géométriques élémentaires. Peu de recherches ont évalué les effets de différents types d'entraînements destinés à favoriser l'apprentissage de la géométrie. Une étude ancienne de G. Prigge (1978) montre l'aide apportée aux enfants par l'introduction de la manipulation. Ainsi, cent quarante six élèves de cinq ans sont assignés à un des trois programmes d'apprentissage des concepts géométriques: activités sans manipulation (test papier-crayon), activités avec manipulation en deux dimensions (pliage de papier, etc.), activités avec manipulation en trois dimensions (solides 
géométriques). Les performances ont été mesurées avant et après dix jours d'activités. Les résultats indiquent que les enfants apprennent mieux les concepts géométriques lorsque des solides géométriques sont disponibles pour chaque élève durant les activités proposées, ceci étant d'autant plus vrai que les élèves ont un niveau de connaissances initial faible.

Plus récemment dans un autre domaine d'apprentissage, des études révèlent les apports bénéfiques de l'exploration visuo-haptique manuelle de lettres en relief dans la préparation à la lecture. É. Gentaz, P. Colé et F. Bara (2003) ont comparé chez des enfants ordinaires de grande section de maternelle (pré-lecteurs) les effets de deux entraînements se différenciant par les modalités sensorielles sollicitées pour appréhender la lettre entraînée, visuo-haptique et haptique pour l'entraînement " HVAM » (HaptiqueVisuel-Auditif-Métaphonologique) et uniquement visuelle pour l'entraînement "VAM » (Visual-AuditifMétaphonologique). Les deux entraînements avaient en commun de proposer des activités sur l'identité du phonème correspondant à la lettre à apprendre. Les enfants ont travaillé six lettres et leurs sons correspondants et ont suivi ces deux entraînements pendant sept semaines (une séance hebdomadaire par lettre et une séance de révision). Les performances ont été mesurées avant et après les entraînements par un test de décodage de pseudo-mots, un test de reconnaissance de lettres et deux tests de conscience phonémique. Les résultats révèlent une amélioration du décodage des pseudo-mots plus importante après un entraînement HVAM qu'après un entraînement VAM. Par ailleurs, une amélioration similaire après les deux entraînements est observée pour les tests d'identification de phonème et de reconnaissance de lettres. L'ajout de la modalité haptique dans ce type d'entraînement semble donc amplifier ses effets bénéfiques seulement sur le niveau de décodage des enfants issus d'écoles ordinaires. Ces résultats ont été confirmés par la suite par d'autres études complémentaires (Bara, Gentaz, Colé \& Sprenger-Charolles, 2004 ; Hillairet de Boisferon, Bara, Gentaz \& Colé, 2007).

Deux explications (non exclusives) peuvent êtres proposées pour expliquer cet effet positif de l'ajout de l'exploration visuo-haptique de lettres en relief. La première est liée aux spécificités fonctionnelles des modalités sensorielles sollicitées (Bara et al., 2004 ; Hatwell, Streri \& Gentaz, 2000). En effet, la séquentialité de l'exploration induite par l'ajout de la modalité haptique pourrait obliger l'enfant à traiter de manière plus séquentielle (et donc plus analytique) les lettres que lorsqu'elles sont présentées visuellement.
L'exploration haptique faciliterait alors le lien entre le traitement visuel de la lettre et le traitement auditif du son correspondant. Cette hypothèse expliquerait pourquoi seules les performances en décodage de pseudo-mots (une mesure de lien son/lettre) sont amplifiées après l'entraînement HVAM. La deuxième hypothèse est liée à l'ajout de la composante motrice inhérente à la modalité haptique. En effet, l'utilisation de l'exploration visuo-haptique dans l'appréhension des lettres pourrait engendrer une meilleure mémorisation de la forme de la lettre car elle serait fondée sur des codages multimodaux (visuel, haptique et moteur). Ce codage multimodal permettrait alors une activation plus rapide des représentations des lettres qui se sont développées à l'aide de multiples sources d'informations (cf. discussion générale pour le soubassement neurophysiologique de ce type de codage). Les résultats observés chez les enfants issus d'écoles ordinaires ne valident pas directement cette hypothèse puisqu'une amélioration similaire apparaît dans le test de reconnaissance de lettre après les deux entraînements.

Cependant, les résultats obtenus chez les enfants scolarisés en zone d'éducation prioritaire ne permettent pas d'exclure cette hypothèse d'un codage multimodal (Bara, Gentaz \& Colé, 2007). Ces enfants qui présentaient des scores initiaux plus faibles que ceux des autres études en vocabulaire, connaissance de lettres et conscience rimique, pouvaient être définis comme des enfants à risque de devenir mauvais lecteurs. Les résultats révèlent une amélioration plus importante du décodage de pseudo-mots après l'entraînement HVAM qu'après l'entraînement VAM mais significative uniquement en CP. En fin de grande section de maternelle, les enfants du groupe HVAM présentent de meilleures performances que ceux du groupe VAM dans les tests d'identification de phonèmes et de reconnaissance de lettres. Cet effet différé de l'entraînement HVAM sur les performances en décodage peut s'expliquer par les faibles performances en vocabulaire et connaissance des lettres présentées par ces enfants en début de grande section. En effet, ces enfants manifestent, avant l'apprentissage formel de la lecture, des retards dans certains domaines langagiers impliqués dans l'acquisition de la lecture. L'exploration haptique, puisqu'elle favorise le lien entre le son et la lettre va permettre d'améliorer les performances des enfants dans deux capacités langagières considérées comme des pré-requis de l'apprentissage: la connaissance de lettres et l'identification de phonèmes. L'exploration haptique faciliterait la mémorisation des lettres et, puisque la connaissance des lettres est interactivement liée à la 
conscience phonémique, on observera alors une amélioration des performances dans ces deux tâches. Le lien entre les lettres et les sons semble d'abord être développé de manière implicite par l'entraînement multisensoriel, puis cette connaissance va être activée et devenir disponible dès le début de l'apprentissage formel de la lecture.

Nous faisons l'hypothèse que les caractéristiques de la modalité haptique - traitement analytique et codage multimodal - pourraient également aider les jeunes enfants à traiter de manière plus efficiente les propriétés géométriques des figures. Ainsi, cette modalité sensorielle pourrait aider les enfants à traiter de la même façon les exemplaires d'une catégorie et donc dépasser leurs spécificités au profit de leur généralité. En d'autres termes, l'introduction de l'exploration visuo-haptique dans le travail sur la reconnaissance des figures élémentaires, compte tenu des caractéristiques spécifiques du système haptique, devrait avoir des effets bénéfiques sur leur reconnaissance. Pour tester cette hypothèse, nous avons proposé deux types d'entraînements respectivement dénommés « visuel ou V » et « visuo-haptique ou $\mathrm{VH}$ » qui se différencient uniquement par les modalités sensorielles sollicitées pour explorer les figures. Ainsi, alors que l'entraînement $V$ sollicite uniquement la modalité visuelle, l'entraînement VH sollicite les modalités visuelle et haptique. Ces deux entraînements ont en commun de proposer des exercices sur les propriétés géométriques de chaque figure. Cependant, ce travail repose sur une exploration des figures uniquement visuelle dans l'entraînement $V$ alors qu'il repose sur une exploration visuelle et visuo-haptique dans l'entraînement VH. Si notre hypothèse est valide, nous devrions observer une amélioration des performances plus importante après l'entraînement VH qu'après l'entraînement V.

\section{MÉTHODE}

\section{Participants}

Trente quatre enfants français âgés en moyenne de cinq ans et six mois (âge réel : de cinq ans à six ans ; écart type : trois mois) ont participé à l'intégralité de cette étude. Ces enfants sont scolarisés dans deux classes de grande section de maternelle d'une école de Grenoble et sont issus de classes socioéconomiques moyennes. Dans chaque classe, les enfants des deux groupes d'entraînement ont été appariés le plus strictement possible sur les critères suivants : âge, effectif, niveau de performance nonverbales (Carrés du WPPSI-R; Weschler, 1995) et niveau de vocabulaire (EVIP ; Dunn, Thériault-Whalen \& Dunn, 1993) présentés dans le tableau I et sur les performances observées aux quatre pré-tests dans le tableau II. II est à noter que quarante six enfants ont participé à cette étude au départ mais douze ont dû être exclus des analyses du fait de leur absence à plus d'une séance d'entraînement. Nous allons voir que ce changement dans les effectifs a eu comme conséquence de modifier l'équivalence des deux groupes. En effet, si la moyenne de bonne reconnaissance des figures cibles dans chaque groupe demeure équivalente (groupe $\mathrm{V}: \mathrm{M}=4,58$ et groupe $\mathrm{VH}: \mathrm{M}=4,27$ ), cela n'est plus le cas pour le nombre moyen de fausses reconnaissances (groupe $V: M=1,27$ et groupe VH : $M=3,30$ ) (cf. tableau 1).

\section{Matériel et procédure}

Les quatre catégories de figures géométriques planes étudiées, le cercle, le carré, le rectangle et le triangle ont été choisies par rapport au contenu du programme de l'Éducation nationale (2002).

Tableau I. - Caractéristiques des enfants des deux groupes avant les entraînements.

Scores moyens (et écart-types) aux tests des Carrés du WPPSI-R, du vocabulaire de I'EVIP, de reconnaissance des figures cibles correctes (max. six) et fausses reconnaissances (max. quatorze)

\begin{tabular}{|l|c|c|c|c|c|c|}
\hline & Effectif & Âge moyen & $\begin{array}{c}\text { Carrés } \\
\text { du WPPSI-R }\end{array}$ & EVIP & Cibles/6 & $\begin{array}{c}\text { Distracteurs } \\
/ 14\end{array}$ \\
\hline Visuel & 17 & $\begin{array}{c}5 \text { ans } \\
\text { et } 5 \text { mois }\end{array}$ & $\begin{array}{c}25,12 \\
(5,2)\end{array}$ & $\begin{array}{c}73,59 \\
(16,35)\end{array}$ & $\begin{array}{c}4,58 \\
(2,40)\end{array}$ & $\begin{array}{c}1,27 \\
(2,40)\end{array}$ \\
\hline Visuo-Haptique & 17 & $\begin{array}{c}5 \text { ans } \\
\text { et } 7 \text { mois }\end{array}$ & $\begin{array}{c}25,06 \\
(6,4)\end{array}$ & $\begin{array}{c}74,58 \\
(13,59)\end{array}$ & $\begin{array}{c}4,27 \\
(3,41)\end{array}$ & $\begin{array}{c}3,30 \\
(2,48)\end{array}$ \\
\hline
\end{tabular}




\section{Pré-tests et post-tests}

Les connaissances géométriques sont mesurées par quatre feuille-tests: une pour chaque catégorie de figure (tests identiques à ceux des annexes $2 \mathrm{a}$ et $2 b$ ). L'ordre des tests est aléatoire. Les enfants ont été évalués environ quinze jours avant (pré-test) et quinze jours après (post-test) les entraînements. Chaque feuille-test contient vingt figures géométriques planes, représentées par leur contour (trait de $0,8 \mathrm{~mm}$ d'épaisseur) sur une feuille de papier (A4 en format paysage). Pour chaque exemplaire de figure, nous avons contrôlé la taille (de $8,5 \mathrm{~cm}$ de côté pour les plus grands à $1,5 \mathrm{~cm}$ de côté pour les plus petits), l'orientation et les rapports entre les côtés. Une feuilletest compte six figures cibles (i.e., six exemplaires de la figure étudiée) et quatorze figures distractrices (i.e., quatorze exemplaires de figures non étudiées). L'ensemble des quatorze figures distractrices d'un test est composé de trois types de figures géométriques, proches perceptivement des propriétés de la figure étudiée. Par exemple, le test du carré contient six exemplaires de carrés et quatorze autres exemplaires de figures, dont des rectangles, des parallélogrammes et des trapèzes, l'ensemble variant en taille et en orientation. Les figures cibles sont numérotées (de un à six) afin de déterminer si certaines sont mieux reconnues que d'autres.

Au-delà des précautions prises dans le choix des figures cibles et distractrices, nous avons évalué la variabilité (distance) entre les figures cibles d'une part et les figures distractrices d'autre part pour les quatre tests. Pour ce faire nous avons associé systématiquement chaque cible à chaque distracteur d'un même test, soit quarante deux associations pour le cercle (1), et quatre vingt quatre pour le carré, quatre vingt quatre pour le rectangle et quatre vingt quatre pour le triangle. L'ensemble des associations est réparti aléatoirement en quatre sous ensembles. Chaque sous-ensemble d'association a été évalué par huit adultes sans consigne de temps. Dans cette épreuve, il est demandé aux sujets de juger sur une échelle de un (très peu ressemblant) à dix (très ressemblant) la ressemblance perceptive entre deux figures. La consigne spécifie de ne juger que sur l'apparence visuelle des figures. Les estimations cumulées du degré de similarité perceptive entre les figures cibles et distractrices données par les adultes pour chaque catégorie de figure sont données dans les annexes $3 \mathrm{a}$ et $3 \mathrm{~b}$. Les résultats montrent que la médiane se situe à cinq pour le cercle, à six pour le carré, à cinq pour le rectangle et à quatre pour le triangle. Ceci signifie que la distance perceptive est jugée comme plus importante pour le triangle, puis viennent le rectangle et le cercle et enfin le carré.

\section{Procédure expérimentale}

Les tests sont administrés par les deux enseignants des classes respectives, de façon individuelle pour éviter que les enfants ne communiquent les réponses. Une formation à la passation des tests a préalablement été proposée aux enseignantes. L'ordre de passation des quatre tests est aléatoire afin de pallier un éventuel effet d'ordre des tests. L'enseignante demande à l'enfant de marquer d'une croix toutes les figures cibles qu'il voit sur sa feuille-test sans lui donner de consigne de vitesse ou de feedback. Enfin, aucun enseignement géométrique n'a été dispensé, depuis le début de l'année scolaire, et ceci, jusqu'aux évaluations finales de cette étude.

\section{Séances d'entraînement}

Deux groupes équivalents sont chacun constitués de dix sept enfants. À chacun des deux groupe d'enfants sélectionnés est attribué un type d'entraînement : un entraînement visuel « $V$ » ou un entraînement visuo-haptique «VH ». Chaque séance d'entraînement, centrée autour de l'étude d'une figure géométrique et de ses propriétés, est proposée chaque semaine aux enfants (cf. encadré 1). Les entraînements se sont déroulés de mars à avril de l'année scolaire. La dernière séance est une révision des propriétés des quatre figures (cf. encadré 2). Les séances se déroulent toujours de la même manière (les mêmes exercices sont effectués dans le même ordre). Chaque séance d'entraînement dure vingt cinq minutes environ et se déroule en salle de classe. Le temps d'entraînement imparti aux séances « V » comme aux séances « VH » est identique. Les enfants, par groupe de cinq ou six, sont assis autour d'une table de manière à favoriser les interactions entre eux. Chaque enfant est sollicité pour participer aux différents exercices. Les deux entraînements se déroulent de la même manière. Cependant, l'entraînement « $V$ » est réalisé avec des figures imprimées sur papier couleur et l'entraînement “ VH " est réalisé avec des figures en relief découpées dans de la mousse (relief de $4 \mathrm{~mm}$ ). Dans les deux cas, les exemplaires de figures proposés sont strictement identiques et collés sur des supports ronds pour ne privilégier aucune orientation. La moitié des figures est de grande taille (huit centimpères sur un support de douze $\mathrm{cm}$ de diamètre) et l'autre moitié est de plus petite taille (quatre centimpères sur un support de sept centimpères de diamètre). 


\section{Encadré 1 : Une séance d'entraînement pour le triangle}

Voici pour exemple, une description détaillée d'une séance qui porte sur le triangle. Dans l'exercice 1, chaque enfant découvre et explore un grand triangle, orienté aléatoirement, l'objectif étant de valider son nom (catégorie) et ses caractéristiques (propriétés avec l'adulte). Dans le groupe d'entraînement « V », les enfants sont invités à regarder attentivement la figure alors que dans le groupe d'entraînement "VH », ils doivent regarder et toucher globalement la figure puis suivre plusieurs fois son contour avec leur index. Une fois que les mouvements d'exploration sont bien maîtrisés par les enfants, l'expérimentateur propose le même exercice mais avec un triangle de plus petite taille (exercice 2). Puis, dans l'exercice 3, l'expérimentateur pose au centre de la table, un grand et un petit triangle pour valider au sein du groupe le concept de triangle en rappelant certaines propriétés, ceci malgré des changements de taille, de couleur et d'orientation qui peuvent intervenir. Ensuite, l'expérimentateur propose une activité avec de grandes cartes figures (exercice 4), faces cachées avec un triangle référent qui reste au centre de la table. Parmi un ensemble de vingt-deux cartes comprenant six triangles et seize figures distractrices, chaque enfant en sélectionne une et la garde face cachée près de lui. L'un après l'autre, chaque enfant retourne et découvre sa grande figure. Après réflexion, il décide s'il s'agit d'un triangle ou non. L'enfant essaie aussi de justifier son choix puis l'adulte valide sa réponse en demandant l'avis au reste du groupe. Puis, l'expérimentateur propose un grand jeu de pioche avec toutes les petites et grandes cartes (exercice 5), faces visibles au centre de la table. L'un après l'autre, chaque enfant doit trouver un exemplaire de triangle parmi d'autres figures distractrices (douze exemplaires de triangles parmi trente deux exemplaires d'autres figures). L'enfant fait son choix, le justifie, le valide ou le corrige éventuellement avec le reste du groupe et l'adulte. Si sa réponse est acceptée, il conserve le triangle sinon il remet la figure sur la table. L'activité se termine lorsque tous les exemplaires de triangles ont été trouvés. Dans cet exercice, la difficulté est plus importante car les figures sont plus nombreuses et la moitié d'entre elles étant plus petite, cela demande une discrimination plus fine. Enfin, l'expérimentateur propose une tâche de classification des figures distractrices restantes (exercice 6). Un enfant choisit une figure distractrice, la décrit aux autres élèves pour qu'ils puissent lui donner tous les exemplaires de cette figure afin de les ranger. Ce déroulement était valable pour les quatre catégories de figures distractrices. L'intérêt est de catégoriser (classement) les figures distractrices en fonction de leurs propriétés mais sans leur attribuer de nom.

\section{Encadré 2 : \\ La séance de révision}

Les enfants revoient les figures géométriques une par une. L'objectif est de réactiver et synthétiser les connaissances acquises par les enfants durant les entraînements. Dans l'entraînement "VH », les enfants rappellent le nom et les propriétés de chaque figure et les explorent manuellement. Dans l'entraînement « V », les figures sont explorées visuellement. Puis les enfants travaillent uniquement avec tous les grands exemplaires des figures cibles (six grands cercles, six grands carrés, six grands rectangles et six grands triangles). La tâche de l'enfant consiste à retourner une figure, à la nommer et à donner ses propriétés. Enfin, la séance se termine avec un grand jeu de pioche de soixante quatre petites et grandes figures: seize cibles (quatre pour chaque figure étudiée, dont deux grandes et deux petites) et quarante huit figures distractrices (déjà utilisées lors des séances d'entraînement).

\section{Analyse descriptive a posteriori des comportements des élèves durant l'étude}

L'analyse descriptive a posteriori des comportements des élèves révèlent tout d'abord que les prétests ne semblent pas poser de problèmes spécifiques. Les consignes sont comprises très rapidement et aisément car aucune question n'est posée par les élèves durant le déroulement des tests. Ensuite, si les séances d'entraînement sur le cercle ne montrent aucune difficulté particulière, celles sur les trois autres figures révèlent des difficultés de vocabulaire: la notion de perpendicularité pour le carré et le rectangle et la confusion entre les termes « rectangle» et « triangle » (probablement due à leur son final commun). Par ailleurs, les enfants reprennent «spontanément " de plus en plus le vocabulaire géométrique approprié et spécifique au domaine employé par l'expérimentateur. Ils deviennent capables d'argumenter leurs réponses en s'appuyant au moins sur une partie des propriétés de la figure étudiée. Enfin, l'orientation spatiale des figures ne semble pas jouer un rôle important puisque certains élèves rapportent pour le post-test sur le carré par exemple: «...que cette 
figure peut être mise n'importe comment, [...] c'est toujours un carré ". De plus, lors des post-tests, certains enfants tournent la feuille-test et/ou vérifient certaines propriétés pour faire leur choix, ce qui n'a jamais été le cas durant les pré-tests.

\section{RÉSULTATS}

Le nombre moyen de figures cibles reconnues et le nombre moyen de fausses reconnaissances pour chaque type de figure avant et après les deux entraînements sont présentés dans le tableau II.

L'analyse du tableau II révèle que le cercle est la figure géométrique la mieux reconnue par les élèves de grande section de maternelle au pré-test avec un score moyen de reconnaissance de 5,94 (soit $99 \%$ ) et un score moyen d'erreur de 0,00 (soit $0 \%$ ) pour les deux entraînements. Ces résultats confirment les résultats de D. Clements et al. (1999) et ceux de L. Pinet et É. Gentaz (2007). Étant donné «l'effet plafond " pour les reconnaissances et "l'effet plancher " pour les erreurs observées avant l'entraînement, les données du cercle ne seront pas intégrées dans les analyses statistiques suivantes.

Dans un premier temps, des corrélations de BravaisPerson ont été réalisées pour évaluer un possible lien entre, d'une part, le score moyen de reconnaissances au pré-test et le score moyen d'erreurs au pré-test et, d'autre part les scores à un test de vocabulaire (EVIP) et aux tests d'aptitude visuo-spatiale (Carrés de la WPPSI-R). Les corrélations entre les scores de vocabulaire avec les scores moyens de reconnaissances $(r=0,03)$ et avec les scores d'erreurs $(r=-0,09)$ ne sont pas significatives $(p>0,25)$. De la même manière, les corrélations entre les scores visuo-spatiaux $(r=0,22)$ et les scores d'erreurs $(r=-0,10)$ ne sont pas significatives $(p>0,25)$. Ces résultats indiquent que ni le niveau de vocabulaire, ni les compétences visuo-spatiales ne sont liées aux performances obtenues aux pré-tests de reconnaissance des figures géométriques (bonnes reconnaissances et erreurs). En conséquence, il n'est pas nécessaire de contrôler ces différents facteurs lors de l'analyse statistique (avec une ANCOVA (2) par exemple). Une ANOVA s'est donc révélée appropriée et suffisante pour analyser nos données.

En conséquence, une ANOVA (2 Entraînement $x$ 2 Période $\times 3$ Figure) sur le score de reconnaissances a été conduite avec pour facteur inter sujet l'Entraînement ( $\mathrm{V}$ ou $\mathrm{VH}$ ) et pour facteurs intra sujet la Période (pré-test et post-test) et la Figure (carré, rectangle et triangle). L'analyse révèle un effet principal de la Figure significatif $[F(2,64)=18,23 ; p<0,001]$ avec $M=4,95$ pour le carré, $M=3,63$ pour le rectangle et $M=3,53$ pour le triangle. En revanche, ni l'Entraînement $[F(1,32)=0,12 ; p>0,25]$ avec $M=3,99$ pour $V$ et $\mathrm{M}=4,09$ pour $\mathrm{VH}$; ni la Période $[\mathrm{F}(1,32)=1,30$;

Tableau II. - Scores moyens (et écart-types) de reconnaissances (max. six) et d'erreurs (max. quatorze) pour les quatre figures avant et après les entraînements visuel (V) et visuo-haptique (VH)

\begin{tabular}{|l|c|c|c|c|c|c|c|c|}
\hline & \multicolumn{4}{|c|}{ Pré-test } & \multicolumn{4}{c|}{ Post-test } \\
\hline & \multicolumn{2}{|c|}{$\begin{array}{c}\text { Cibles } \\
\text { reconnues }\end{array}$} & \multicolumn{2}{c|}{$\begin{array}{c}\text { Fausses } \\
\text { reconnaissances }\end{array}$} & \multicolumn{2}{c|}{$\begin{array}{c}\text { Cibles } \\
\text { reconnues }\end{array}$} & \multicolumn{2}{c|}{$\begin{array}{c}\text { Fausses } \\
\text { reconnaissances }\end{array}$} \\
\hline $\begin{array}{l}\text { Entraînement/ } \\
\text { Figure }\end{array}$ & $\mathrm{V}$ & $\mathrm{VH}$ & $\mathrm{V}$ & $\mathrm{VH}$ & $\mathrm{V}$ & $\mathrm{VH}$ & $\mathrm{V}$ & $\mathrm{VH}$ \\
\hline Cercle & $\begin{array}{c}5,94 \\
(0,24)\end{array}$ & $\begin{array}{c}5,94 \\
(0,24)\end{array}$ & $\begin{array}{c}0,00 \\
(0,00)\end{array}$ & $\begin{array}{c}0,00 \\
(0,00)\end{array}$ & $\begin{array}{c}5,76 \\
(0,56)\end{array}$ & $\begin{array}{c}5,94 \\
(0,24)\end{array}$ & $\begin{array}{c}0,00 \\
(0,00)\end{array}$ & $\begin{array}{c}0,00 \\
(0,00)\end{array}$ \\
\hline Carré & $\begin{array}{c}4,88 \\
(1,36)\end{array}$ & $\begin{array}{c}4,88 \\
(1,54)\end{array}$ & $\begin{array}{c}1,41 \\
(1,97)\end{array}$ & $\begin{array}{c}4,59 \\
(2,50)\end{array}$ & $\begin{array}{c}4,71 \\
(1,65)\end{array}$ & $\begin{array}{c}5,35 \\
(1,17)\end{array}$ & $\begin{array}{c}4,71 \\
(2,11)\end{array}$ & $\begin{array}{c}2,94 \\
(2,86)\end{array}$ \\
\hline Rectangle & $\begin{array}{c}4,47 \\
(1,12)\end{array}$ & $\begin{array}{c}3,06 \\
(2,14)\end{array}$ & $\begin{array}{c}1,65 \\
(2,32)\end{array}$ & $\begin{array}{c}3,06 \\
(1,95)\end{array}$ & $\begin{array}{c}3,47 \\
(1,87)\end{array}$ & $\begin{array}{c}3,53 \\
(1,91)\end{array}$ & $\begin{array}{c}2,06 \\
(2,11)\end{array}$ & $\begin{array}{c}3,35 \\
(2,23)\end{array}$ \\
\hline Triangle & 3,06 & $\begin{array}{c}3,24 \\
(1,39)\end{array}$ & $\begin{array}{c}2,06 \\
(3,53)\end{array}$ & $\begin{array}{c}5,59 \\
(4,44)\end{array}$ & $\begin{array}{c}3,35 \\
(0,86)\end{array}$ & $\begin{array}{c}4,47 \\
(1,18)\end{array}$ & $\begin{array}{c}0,24 \\
(0,56)\end{array}$ & $\begin{array}{c}0,59 \\
(1,94)\end{array}$ \\
\hline
\end{tabular}


$p>0,25]$ avec $M=3,93$ aux pré-tests et $M=4,15$ aux post-tests, n'ont un effet. L'interaction Entraînement $\times$ Figure est significative $[F(2,64)=3,43 ; p<0,05]$ avec pour le carré $M=4,79$ pour $V$ contre $M=5,11$ pour $\mathrm{VH}$, pour le rectangle $\mathrm{M}=3,97$ pour $\mathrm{V}$ contre $M=3,29$ pour $V H$, et pour le triangle $M=3,20$ pour $\mathrm{V}$ contre $\mathrm{M}=3,85$ pour $\mathrm{VH}$. L'interaction Période $\times$ Figure est également significative $[F(2,64)=3,62$; $\mathrm{p}<0,05]$ avec aux pré-tests, $\mathrm{M}=4,88$ pour le carré, $M=3,76$ pour le rectangle et $M=3,15$ pour le triangle, contre aux post-tests, $M=5,03$ pour le carré, $M=3,50$ pour le rectangle et $M=3,91$ pour le triangle. L'interaction Entraînement $\times$ Période est significative $[F(1,32)=7,31 ; p<0,05]$. Pour examiner notre hypothèse sur l'effet de l'exploration haptique, nous avons réalisé deux comparaisons planifiées, entre le pré-test $(M=4,13)$ et le post-test $(M=3,84)$ pour l'entraînement « $V$ » et entre le pré-test $(M=3,72)$ et le post-test $(M=4,45)$ pour l'entraînement " $V H$ ». Ces deux résultats indiquent que l'entraînement «VH » permet une progression significative entre le pré-test et le post-test $[F(1,32)=7,40 ; p<0,05]$, ce qui n'est pas le cas pour l'entraînement « $V$ » $[F(1,32)$ $=1,21 ; p>0,25]$. Ce résultat va dans le sens de notre hypothèse selon laquelle l'entraînement visuo-haptique serait plus efficace que l'entraînement visuel du point de vue du nombre de figures cibles reconnues. Enfin, l'interaction entre les facteurs Entraînement $\times$ Période $\times$ Figure n'est pas significative $[F(2,64)=$ $0,59 ; p>0,25]$

D'autre part, nous avons déjà signalé que si le score reconnaissance est équivalent entre les deux groupes ( $M=4,58$ pour $V$ et $M=4,28$ pour $V H$ ), cela n'est plus le cas pour le score moyen d'erreurs $(M=1,28$ pour $V$ et $\mathrm{M}=3,31$ pour $\mathrm{VH}$ ). Pour prendre en compte cette différence initiale entre les deux groupes concernant le nombre d'erreurs (cf. tableau I), nous avons calculé un indice de progression pour chaque figure entre le pré-test et le post-test. Un indice positif signifie que l'enfant a progressé faisant moins d'erreurs après l'entraînement qu'avant alors qu'un indice négatif signifie le contraire. Ainsi, une ANOVA (2 Entraînement $\times 3$ Figure) sur cet indice de progression a été conduite avec pour facteur inter sujets l'Entraînement ( $\mathrm{V}$ ou $\mathrm{VH}$ ) et pour facteur intra sujet la Figure (carré, rectangle et triangle). Cette analyse indique un effet significatif de l'entraînement $[F(1,32)=8,83$; $p<0,001]$, avec un progrès général plus important pour le groupe $\mathrm{VH}(\mathrm{M}=+2,11)$ que pour le groupe $\mathrm{V}(\mathrm{M}=-0,62)$. Un effet significatif ressort aussi pour la Figure $[F(2,64)=40,20 ; p<0,001]$ avec une progression plus importante pour le triangle $(\mathrm{M}=+3.41)$ comparativement au rectangle $(\mathrm{M}=-0.35)[\mathrm{F}(1,32)$
$=47,50 ; \mathrm{p}<0,001]$ ou au carré $(\mathrm{M}=-0.82)[\mathrm{F}(1$, 32) $=71,84 ; p<0,001]$; autrement dit, ce sont pour les triangles que les entraînements sont les plus efficaces, en permettant de diminuer les erreurs des enfants. Enfin, l'interaction Entraînement $\times$ Figure est significative $[F(2,64)=11,10 ; p<0,001]$. Pour spécifier les apports bénéfiques de la modalité haptique, nous avons comparé les effets des entraînements pour chacune des trois figures. II ressort que l'entraînement VH est plus bénéfique que l'entraînement $\mathrm{V}$ pour le carré (groupe $\mathrm{V}=-3,29$ et groupe $\mathrm{VH}$ $=+1,64)[F(1,32)=19,79 ; p<0,001]$ et le triangle (groupe $\mathrm{V}=+1,82$ et groupe $\mathrm{VH}=+5,00)[\mathrm{F}(1,32)=$ $6,23 ; p<0,05]$, ce qui n'est pas le cas pour le rectangle (groupe $\mathrm{V}=+0,41$ et groupe $\mathrm{VH}=+0,29)[\mathrm{F}(1,32)$ $=0,17 ; p=0,25]$. Ces résultats confortent les résultats obtenus avec les reconnaissances en montrant que l'ajout de la modalité haptique dans ce type d'entraînement améliore également les performances des enfants en diminuant les erreurs commises pour le carré et le triangle par rapport au groupe contrôle V.

\section{DISCUSSION}

Cette recherche vise à améliorer l'apprentissage des figures géométriques élémentaires et plus particulièrement, à savoir si l'ajout de la modalité haptique dans un entraînement destiné à préparer cet apprentissage est bénéfique pour les enfants de grande section de maternelle. Pour cela, nous avons comparé deux entraînements destinés à développer les connaissances géométriques se différenciant par les modalités sensorielles sollicitées pour explorer les figures. Nous avons mesuré les performances avant et après les entraînements au moyen de quatre tests de reconnaissances de figures cibles parmi des distracteurs. Ces quatre épreuves permettent de recueillir deux types de données : le nombre de bonnes reconnaissances (figures cibles cochées) et le nombre d'erreurs (figures distractrices cochées) pour chacune des quatre figures étudiées (cercle, carré, rectangle et triangle).

Les résultats montrent que le nombre moyen de figures cibles correctement reconnues progresse significativement après l'entraînement visuo-haptique, résultat qui n'est pas observé après l'entraînement visuel. En d'autres termes, seul l'entraînement visuohaptique permet aux enfants de mieux reconnaître les figures cibles à l'issue des séances d'entraînement. D'autre part, l'analyse des erreurs révèle que 
les enfants progressent en commettant moins d'erreurs pour le carré et le triangle suite à l'entraînement $\mathrm{VH}$. Ces deux analyses indiquent aussi que toutes les figures ne profitent pas de la même façon des entraînements. En effet, l'entraînement VH permet de mieux catégoriser les carrés (plus de reconnaissances et moins d'erreurs suite à cet entraînement), ce qui n'est pas le cas après l'entraînement visuel. Pour le rectangle, les deux entraînements ne permettent pas vraiment aux enfants de mieux cerner cette catégorie. Enfin, c'est pour le triangle que les entraînements semblent les plus efficaces, en augmentant les reconnaissances suite à l'entraînement $\mathrm{VH}$ et en diminuant les erreurs après chacun des deux entraînements, mais ceci de façon plus importante après l'entraînement VH. Le niveau initial du cercle semble quant à lui trop élevé pour que l'on puisse observer une différence entre les deux entraînements. Ces différences entre les figures, indépendamment des entraînements, peuvent s'expliquer notamment par la quantité de propriétés nécessaires à traiter pour identifier les différentes figures (côtés et sommets pour le triangle ; côtés, sommets, longueurs équivalentes et notion de perpendicularité pour le carré ; côtés, sommets, longueurs équivalentes deux à deux et notion de perpendicularité pour le rectangle) et par le niveau initial de connaissances. Voyons maintenant comment le traitement de ces propriétés pourrait différer selon l'entraînement.

L'ajout de la modalité haptique dans ce type d'entraînement apporte des effets bénéfiques en permettant aux enfants de mieux cerner ce qui est inclus et exclu dans une catégorie de figure. Deux interprétations peuvent rendre compte de ces effets bénéfiques. D'une part, percevoir une figure implique des processus de traitement beaucoup plus analytiques et séquentiels de ces propriétés lorsqu'elle est explorée avec la modalité haptique qu'avec la modalité visuelle, et ce plus particulièrement à cinq-six ans (Berger \& Hatwell, 1993, 1995, 1996). Ainsi, les explorations visuelle et haptique des figures aideraient les jeunes enfants à davantage dissocier et traiter les propriétés géométriques spécifiques à chaque catégorie de figures que l'exploration seulement visuelle. D'autre part, et de façon non incompatible avec l'interprétation précédente, l'utilisation de l'exploration haptique dans l'appréhension des figures pourrait engendrer une meilleure mémorisation et reconnaissance de leur forme. L'exploration haptique des figures implique un codage multimodal en mémoire à la fois visuel, haptique et moteur. Ce codage multimodal permettrait alors une meilleure activation des représentations des figures qui se sont développées à l'aide de multiples sources d'informations. Les tests de reconnaissance des figures utilisés dans la présente expérience pourraient constituer une mesure globale de cette connaissance qui doit prendre en compte la reconnaissance des figures mais également la rapidité à les reconnaître. La rapidité d'activation des représentations des figures n'a pas été évaluée dans ces études et des recherches complémentaires sont donc nécessaires pour tester cette hypothèse.

Quelques travaux connexes en imagerie cérébrale fonctionnelle chez l'adulte sur la représentation corticale des objets et des lettres permettent de mieux comprendre ces effets bénéfiques. Ainsi, L. Chao et A. Martin (2000) ont montré que la présentation visuelle d'objets qui sont associés à une action spécifique, active une zone dans le cortex pré-moteur, même si aucune réponse motrice n'est requise. La connaissance sensorimotrice des propriétés fonctionnelles des objets manipulables serait une partie intégrante de leur représentation, et pourrait être utilisée pour les reconnaître. Par ailleurs, M. Longcamp, J.-L. Anton et J.-L. Velay (2003) montrent que la présentation visuelle de lettres mais pas celle de pseudo-lettres, active une zone dans le cortex prémoteur gauche, alors qu'aucune réponse motrice n'est requise. Cette zone est activée à la fois pour la vision de la lettre et pour son écriture. Ces résultats suggèrent que la reconnaissance visuelle des lettres est sous tendue par une représentation corticale multimodale dont l'une des composantes serait de nature sensorimotrice. II est possible que de tels mécanismes puissent être mis en jeu lors de l'apprentissage des figures géométriques.

Discutons maintenant de ces résultats par rapport au modèle proposé par D. van Hiele (van Hiele, 1959 ; van Hiele, 1986 ; van Hiele-Geldof, 1984). Dans ce dernier, les auteurs proposent que la pensée géométrique des élèves évolue selon une progression en cinq stades successifs. Seuls les premiers stades nous intéressent ici. Ainsi, au cours du premier niveau (visualisation), les élèves perçoivent les objets géométriques en fonction de leur apparence perceptive, sans utiliser explicitement leurs propriétés géométriques. Par exemple, les élèves considèrent qu'un triangle est un triangle parce "sa pointe est en haut ". Ce niveau ne semble pas facile à dépasser car M. Shaughnessy et W. Burger (1985) ont montré que plus de $70 \%$ des élèves entrant au lycée sont au niveau 1, et que seuls les étudiants qui parviendront à atteindre le niveau 2 auront une chance d'arriver ultérieurement à justifier leur raisonnement. Le second niveau (analytique) est celui de l'analyse. Les élèves 
sont capables d'associer les objets géométriques à leurs propriétés en utilisant une séquence de propriétés nécessaires pour les identifier. Par exemple, les élèves considèrent qu'un carré est un carré parce qu'il possède d'abord quatre côtés de même longueur, puis quatre angles droits et enfin que ses côtés opposés sont parallèles. Selon les auteurs, l'avancement d'un niveau à l'autre dépendrait du contenu et du mode d'instruction, et chaque niveau possèderait son propre vocabulaire et son propre système de relations. Des recherches récentes affinent le modèle de D. van Hiele en montrant par exemple l'existence d'un niveau "pré cognitif " avant le niveau 1 (visualisation). Par exemple, D. Clements et ses collègues (1999) montrent que les jeunes enfants forment des "schémas initiaux" sur la base de l'analyse des caractéristiques des figures visuelles. Tandis que ces schémas se développent, les enfants continuent à compter principalement sur l'apparence visuelle pour distinguer des figures. Ils sont, cependant, également capables d'identifier quelques propriétés simples des figures familières. Ainsi, les auteurs montrent l'existence d'un niveau pré cognitif (niveau 0) avant le niveau 1. Au niveau 0 , les enfants percevraient des figures géométriques, mais peut-être en raison d'une insuffisance dans l'activité perceptive (Piaget \& Inhelder, 1948), pourraient seulement s'occuper des caractéristiques visuelles d'une figure et ainsi ne pas identifier beaucoup de figures communes. Les résultats de la présente expérience confirment que la nature des contenus et des méthodes proposées aux enfants sont cruciaux pour l'acquisition de la reconnaissance des figures géométriques.

En conclusion, l'ensemble des résultats permet de confirmer que, même si des connaissances géométriques existent chez les jeunes enfants, un apprentissage incluant la modalité haptique leurs sont nécessaire et bénéfique pour mieux appréhender les propriétés essentielles de chaque catégorie de figures élémentaires.

Laëtitia Pinet laetitia@editions-cigale.com

Édouard Gentaz Edouard.gentaz@upmf-grenoble.fr

CNRS, Laboratoire de psychologie et neurocognition ", UMR 5105, université Pierre-Mendès-France-Grenoble 2

\section{REMERCIEMENTS}

Nous remercions tout particulièrement, Blandine Guillemet et Sonia Rumillat, enseignantes de grande section à l'école Jean Jaurès de Grenoble pour leur motivation et implication dans cette étude, ainsi que la directrice, Catherine Gilloux. Nous remercions évidemment les élèves pour leur participation ainsi que leurs parents qui nous ont fait confiance. Merci aussi, à l'université Pierre-Mendès-France-Grenoble 2, au CNRS et aux Éditions la Cigale pour leur soutien.
(1) Le test de similarité perceptive du cercle compte quarante deux associations puisque qu'il n'y a aucune différence perceptive lorsqu'un même cercle est orienté différemment (ce qui n'est pas le cas pour le carré, le rectangle et le triangle).
(2) Si des variables, autres que celles manipulées (niveau de vocabulaire ou aptitudes spatiales), se révèlent être liées (i.e., par des corrélations positives) aux variables mesurées (i.e., bonnes reconnaissances ou erreurs), ces premières variables doivent être prise en compte dans le traitement des données en utilisant une analyse nommée ANCOVA (Dancey, Reidy \& Gauvrit, 2007).

\section{BIBLIOGRAPHIE}

BARA F., GENTAZ É., COLÉ P. \& SPRENGER-CHAROLLES L. (2004). "The visuo-haptic and haptic exploration of letters increases the kindergarten children's understanding of the alphabetical principle ". Cognitive Development, $\mathrm{n}^{\circ} 19$, p. 433-449.
BARA F., GENTAZ É. \& COLÉ P. (2007). «The visuo-haptic and haptic exploration of letters increases the decoding level of children coming from low-socioeconomic status families ». British Journal of Developmental Psychology. $n^{\circ} 25$, p. 643-663. 
BARUK, S. (1995). Dictionnaire de mathématiques élémentaires. Paris : Seuil.

BERGER C. \& HATWELL Y. (1993). « Dimensionnal and overall similarity classifications in haptics: A developmental study ». Cognitive Development, n 8, p. 495-516.

BERGER C. \& HATWELL Y. (1995). «Development of analytic vs. global processing in haptics: The perceptual and decisional determinants of classification skills". British Journal of Developmental Psychology, $\mathrm{n}^{\circ} 13$, p. 143-162.

BERGER C. \& HATWELL Y. (1996). " Developmental trends in haptic and visual free classifications: Influence of stimulus structure and exploration on decisional processes". Journal of Experimental Child Psychology, $n^{\circ} 63$, p. 447-465.

CHAO L. \& MARTIN A. (2000). "Representation of manipulable man-made objects in the dorsal stream ". Neuroimage, $\mathrm{n}^{\circ} 12$, p. 478-484.

CLEMENTS D., SWAMINATHAN S., HANNIBAL M. \& SARAMA J. (1999). "Young children's concepts of shape ". Journal for Research in Mathematics Education, $n^{\circ} 30$, p. 192-212.

DANCEY C., REIDY, J. \& GAUVRIT N. (2007). Statistiques sans maths pour psychologies. Bruxelles: De Boeck.

DUNN L., THERIAULT-WHALEN C. \& DUNN L. (1993). Échelle de vocabulaire en images Peabody. Toronto: Éditions Psycan.

FRANCE : MINISTÈRE DE L'ÉDUCATION NATIONALE (2003). Évaluations $C E 2-6^{e}-5^{e}$. Repères nationaux, septembre 2002. Direction de l'évaluation et de la prospective.

FRANCE : MINISTÈRE DE L'ÉDUCATION NATIONALE (2002). Qu'apprend-on à l'école élémentaire? Les nouveaux programmes. Paris : CNDP, XO Editions.

GENTAZ É., COLE P. \& BAR F. (2003). «Évaluation d'entraînements multisensoriels de préparation à la lecture pour les jeunes enfants de grande section de maternelle : une étude sur la contribution du système haptique manuel ». L'Année Psychologique, $\mathrm{n}^{\circ} 104$, p. 561-584.

HATWELL Y., STRERI A. \& GENTAZ É (2000). Toucher pour connaitre. Psychologie cognitive de la perception tactile manuelle. Paris : PUF.
HILLAIRET DE BOISFERON A., GENTAZ É \& COLÉ P. (2007). "Préparation à la lecture des jeunes enfants : Effets de l'exploration visuo-haptique des lettres et de la perception visuelle des mouvements d'écriture ". L'Année Psychologique, $\mathrm{n}^{\circ} 7$, p. 537-564.

HULME C. (1981). Reading retardation and multisensory teaching. Londres: Routledge \& Kegan Paul.

LONGCAMP M., ANTON JL. \& VELAY JL. (2003). "Visual presentation of single letters activates a premotor area involved in writing ». Neuroimage, $n^{\circ} 19$, p. 1492-1500.

NEISSER U. (1987). Introduction : The ecological and intellectual bases of categorization. In U. Neisser (éd.), Concepts and conceptual development: Ecological and intellectual factors in categorisation. Cambridge : Cambridge University Press.

PIAGET J. \& INHELDER B. (1948). La représentation de l'espace chez l'enfant. Paris : PUF.

PINET I. \& GENTAZ É. (2007). «La reconnaissance de figures géométriques planes (cercle, carré, rectangle et triangle) chez les enfants de 5 ans . Grand N, $\mathrm{n}^{\circ} 80$, p. 17-28.

PRIGGE G. (1978). "The differential effects of the use of manipulative aids on the learning geometric concepts by elementary school children ». Journal for Research in Mathematics Education, n 9, p. 361-367.

SHAUGHNESSY M. \& BURGER W. (1985). "Spadework prior to deduction in geometry ". Mathematics Teacher, $n^{\circ} 78$, p. $419-428$.

VAN HIELE P. \& M. (1959). " Development and learning process ». Acta Paedagogica Ultrajectina, $\mathrm{n}^{\circ} 17$, p. 2-31.

VAN HIELE P. \& M. (1986). Structure and insight. Orlando: Academic Press.

VAN HIELE-GELDOF D. (1984). The didactics of geometry in the lowest class of secondary school. In D. Fuys, D. Geddes, \& R. Tischler (Eds.), English translation of selected writings of Dina van Hiele-Geldof and Pierre M. van Hiele (p. 1-214). Brooklyn, New-York: Brooklyn College, School of Education. (ERIC Document Reproduction Service $\mathrm{n}^{\circ} 289697$ ).

WECHSLER D. (1995). Échelle d'Intelligence de Wechsler pour la période PréScolaire et Primaire: forme révisée (WPPSI-R manuel). Paris : Éditions ECPA. 
Annexe 1. - Les quatre tests proposés aux enfants par Clements et al. (1999)

1. Student marks circles (Razel \& Eylon, 1991).

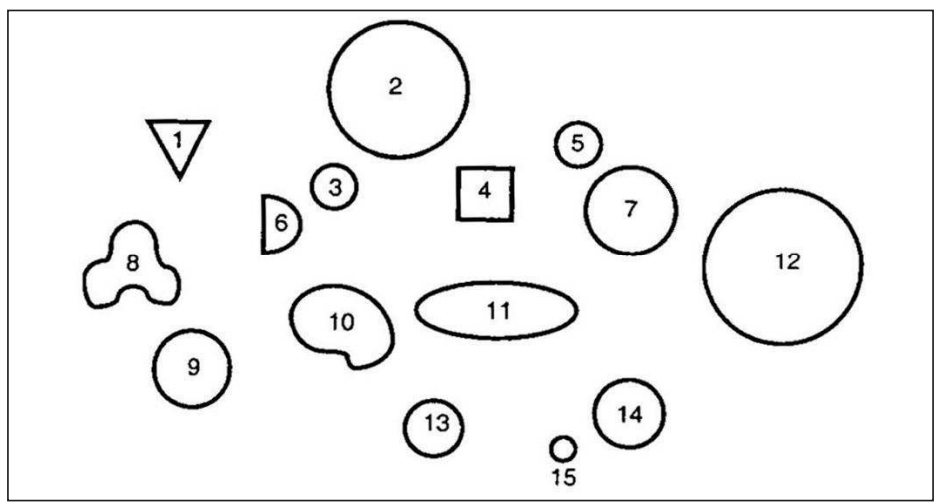

2. Student marks squares (Razel \& Eylon, 1991).

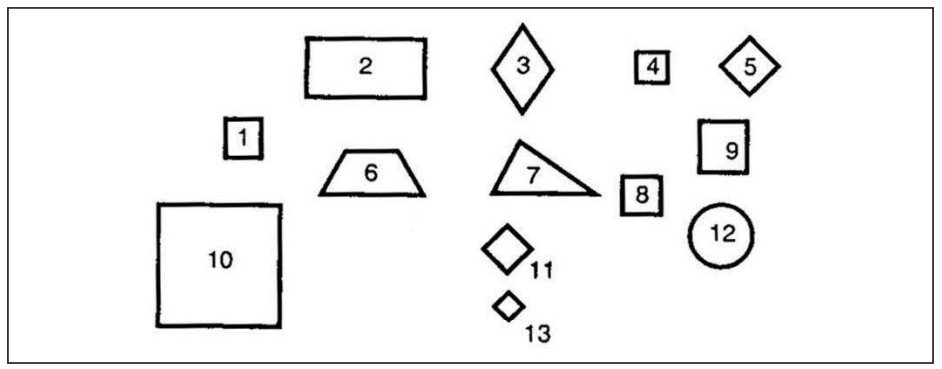

3. Student marks triangles (Burger \& Shaughnessy, 1986 ; Clements

4. Student marks rectangles (Burger \& Shaughnessy, 1986 ; Clements \& Battista, 1992a).
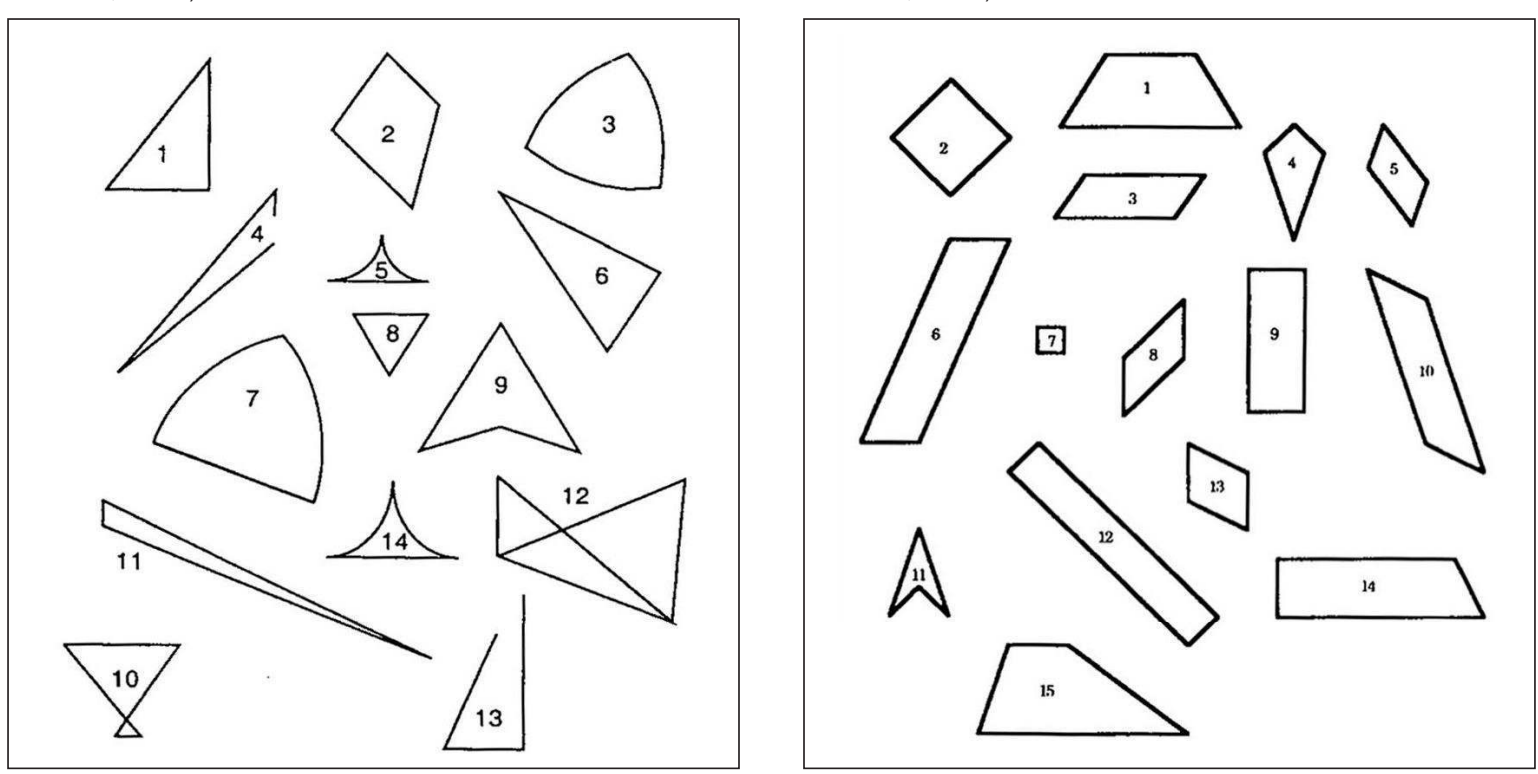
Annexe 2a. - Les tests du cercle et du carré proposés aux enfants par Pinet \& Gentaz (2007)
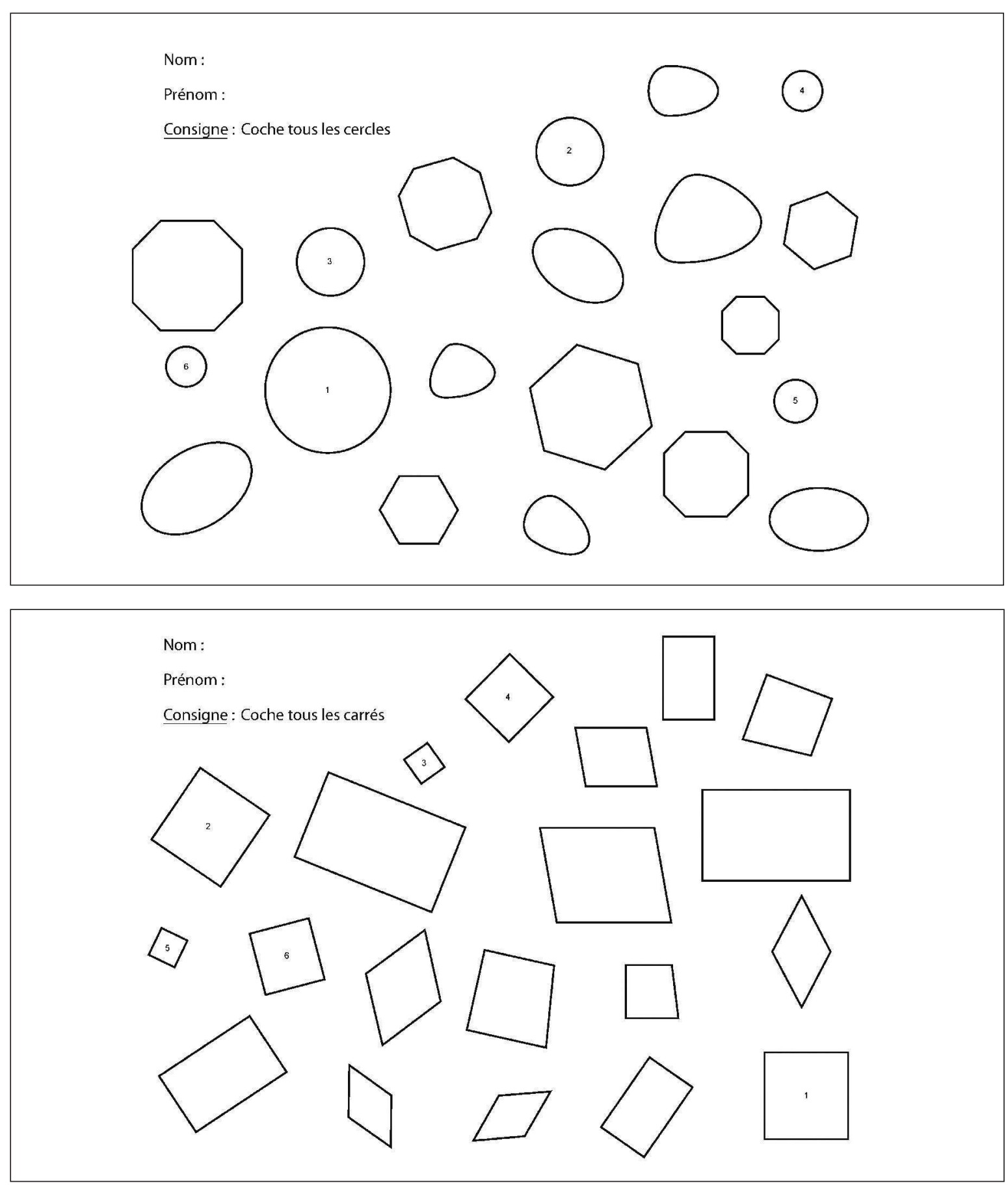

Évaluation d'entraînements multisensoriels de préparation à la reconnaissance de figures géométriques planes chez les enfants... 
Annexe $2 b$ - Les tests du rectangle et du triangle proposés aux enfants par Pinet \& Gentaz (2007)
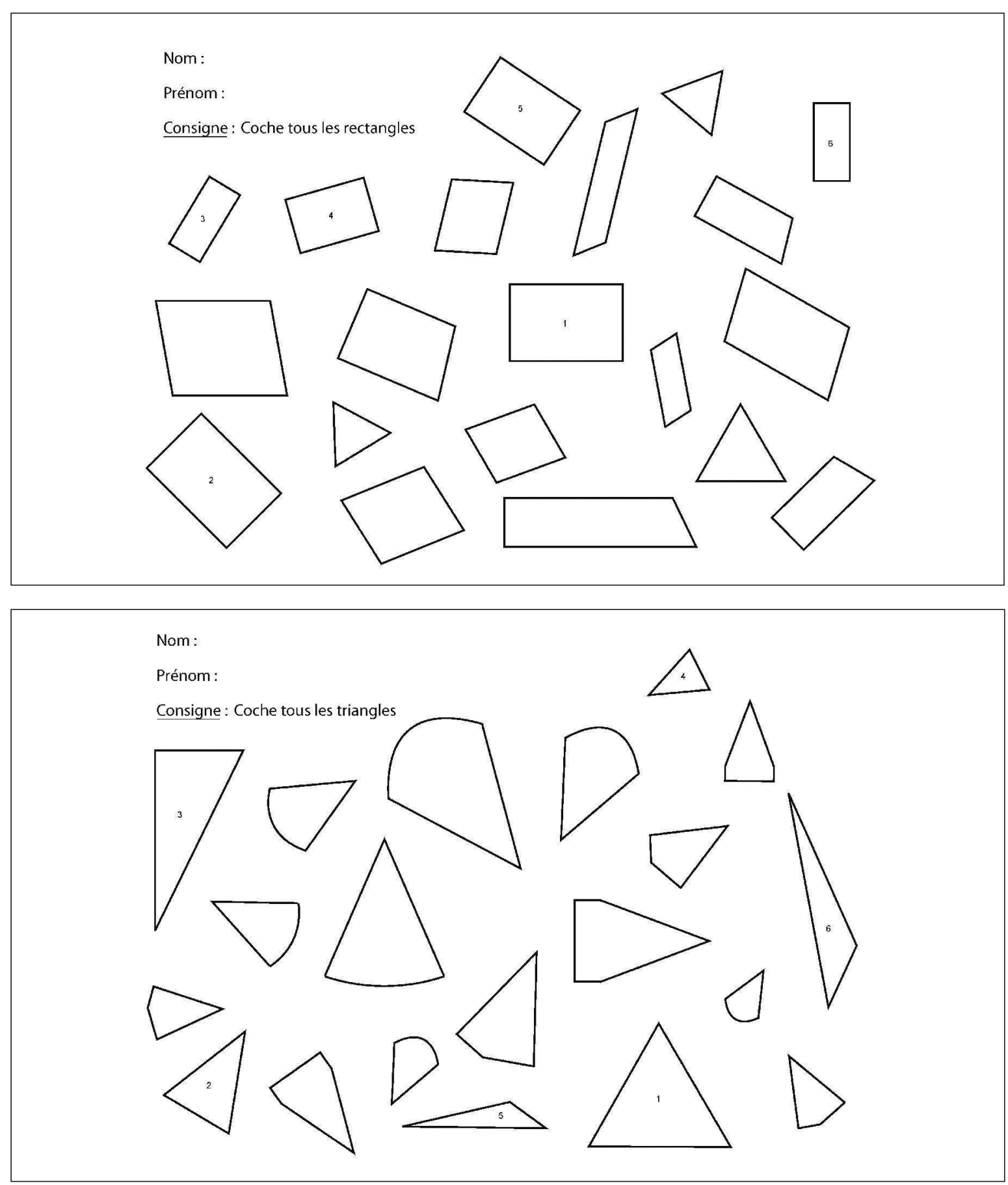

Revue française de pédagogie | 162 | janvier-février-mars 2008 
Annexe 3a. - Estimations cumulées du degré de similarité perceptive entre les figures cibles et les figures distractrices données par des adultes pour le cercle et le carré

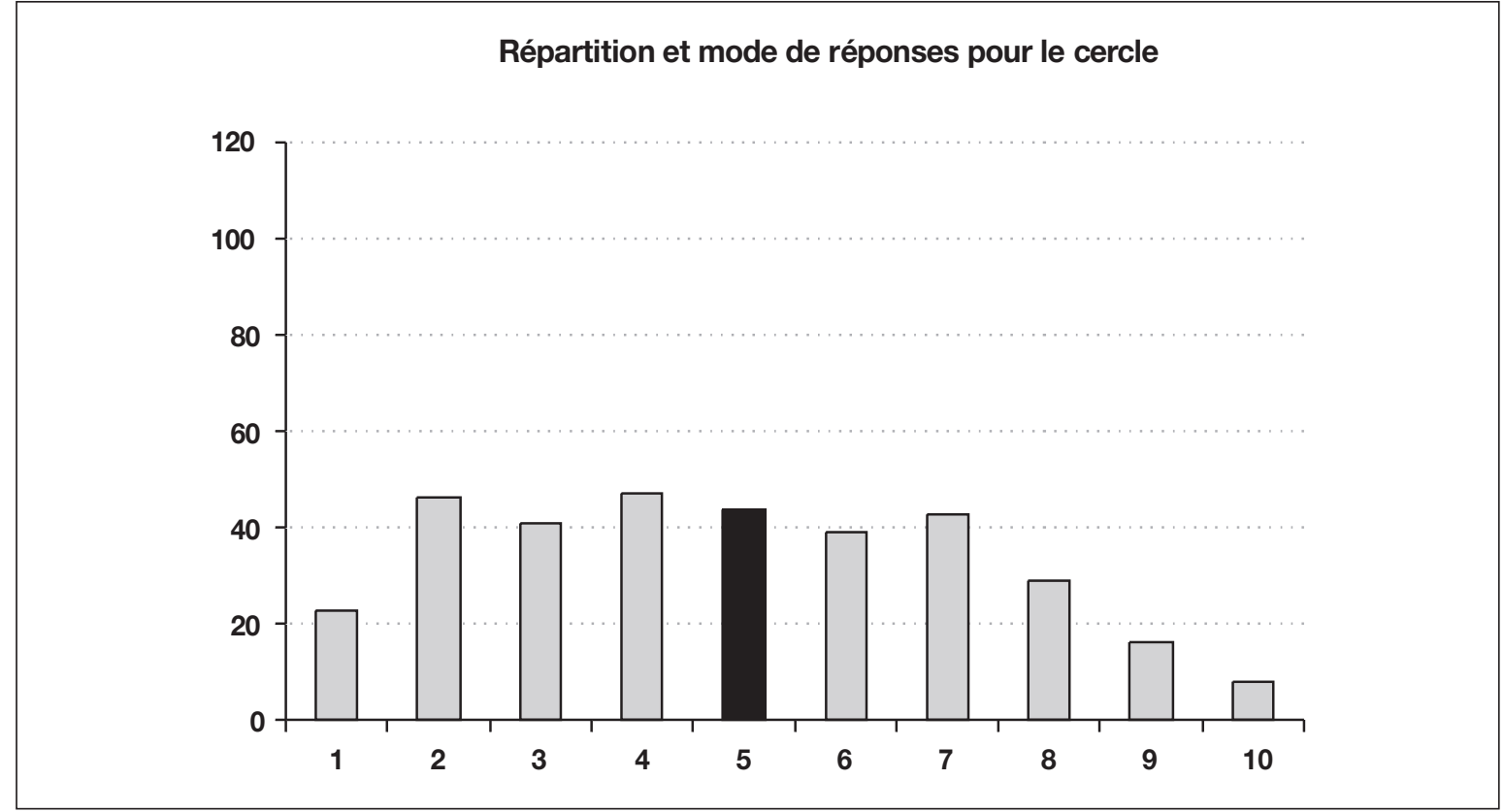

Notes : échelle de 1 (très peu ressemblant) à 10 (très ressemblant) par pas de 1 ; la réponse médiane est indiquée en noire.

Répartition et mode de réponses pour le carré

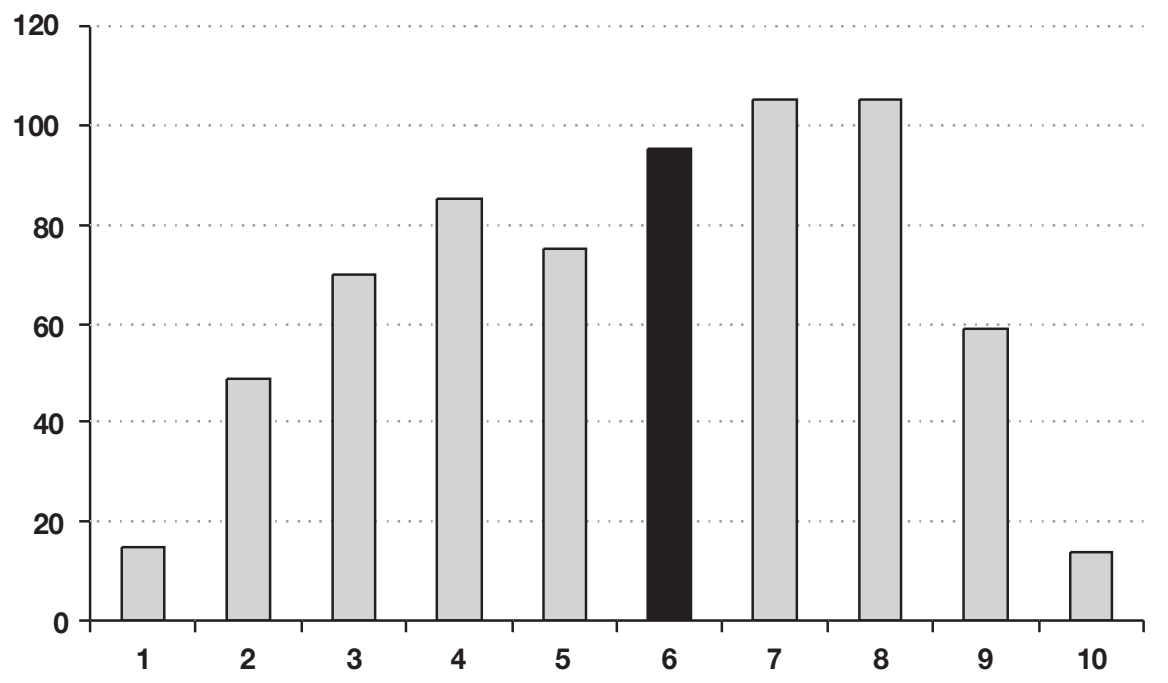


Annexe 3b. - Estimations cumulées du degré de similarité perceptive entre les figures cibles et les figures distractrices données par des adultes pour le rectangle et le triangle

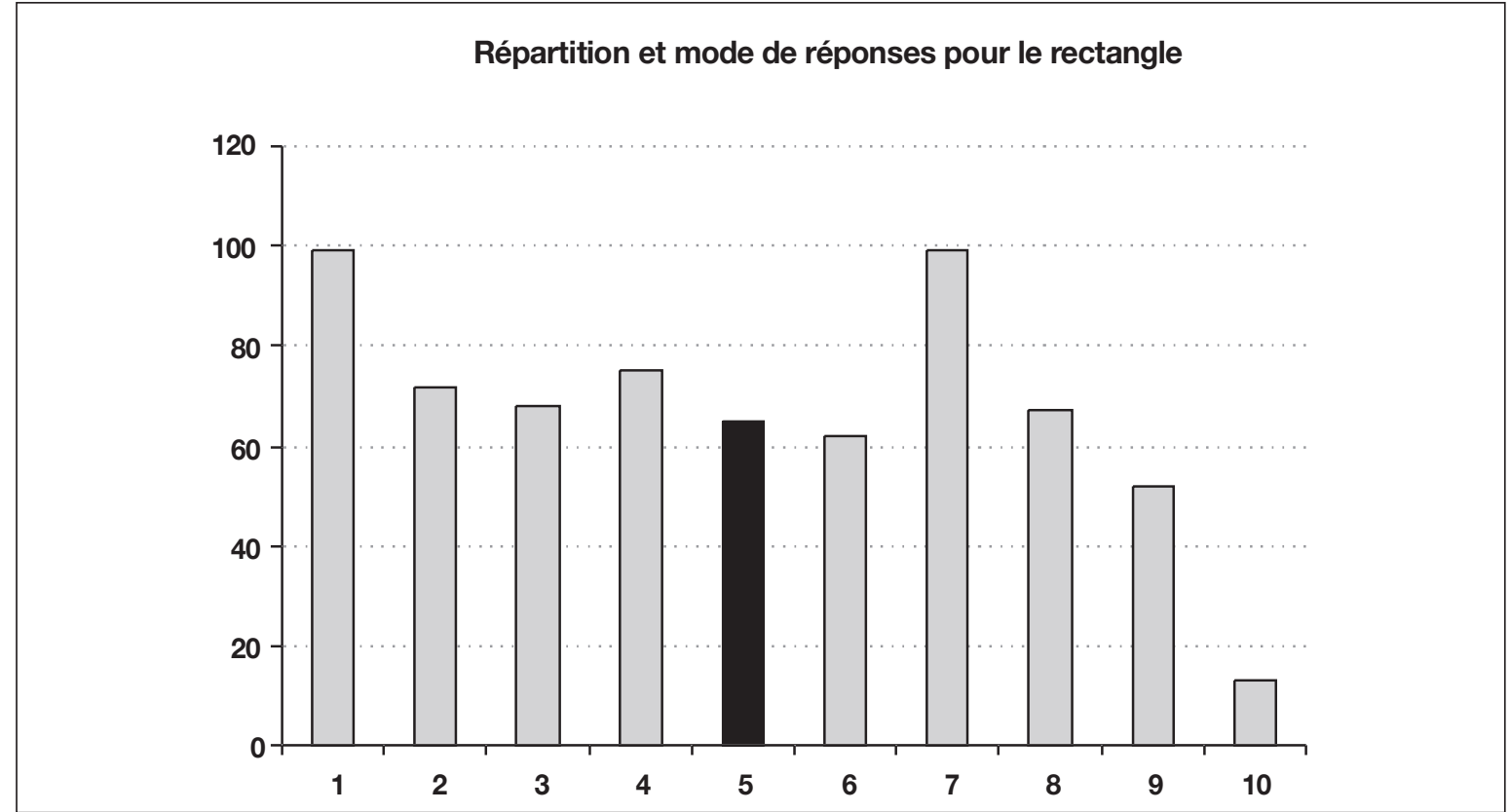

Notes : échelle de 1 (très peu ressemblant) à 10 (très ressemblant) par pas de 1 ; la réponse médiane est indiquée en noire.

Répartition et mode de réponses pour le triangle

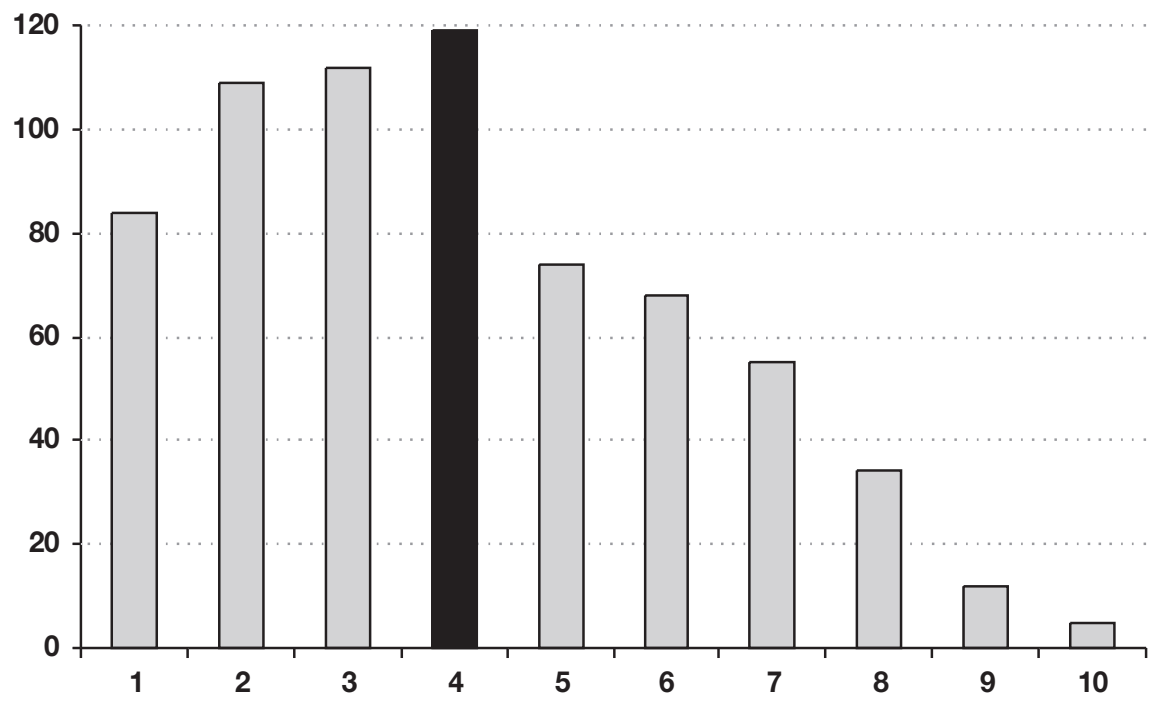

